\title{
GRB 070201: A POSSIBLE SOFT GAMMA-RAY REPEATER IN M31 ${ }^{1}$
}

\author{
E. O. Ofek, ${ }^{2}$ M. Muno, ${ }^{2}$ R. Quimby, ${ }^{2}$ S. R. Kulkarni, ${ }^{2}$ H. Stiele, ${ }^{3}$ W. Pietsch, ${ }^{3}$ E. Nakar, ${ }^{2}$ \\ A. Gal-Yam, ${ }^{4}$ A. Rau, ${ }^{2}$ P. B. Cameron, ${ }^{2}$ S. B. Cenko, ${ }^{2}$ M. M. Kasliwal, ${ }^{2}$ \\ D. B. Fox, ${ }^{5}$ P. Chandra, ${ }^{6,7}$ A. K. H. Kong, ${ }^{8,9}$ and R. Barnard ${ }^{10}$ \\ Received 2007 December 13; accepted 2008 February 18
}

\begin{abstract}
GRB 070201 was a bright, short-duration, hard-spectrum gamma-ray burst detected by the Interplanetary Network. Its error quadrilateral, which has an area of $0.124 \mathrm{deg}^{2}$, intersects some prominent spiral arms of the nearby M31 (Andromeda) galaxy. Given the properties of this GRB, along with the fact that LIGO data argue against a compact binary merger origin in M31, it is an excellent candidate to have been an extragalactic soft gamma-ray repeater (SGR) giant flare, with an energy of $1.4 \times 10^{45} \mathrm{ergs}$. However, we cannot rule out the possibility that it was a short-duration GRB in the background. Analysis of ROTSE-IIIb visible-light observations of M31, taken $10.6 \mathrm{hr}$ after the burst and covering $42 \%$ of the GRB error region, does not reveal any optical transient down to a limiting magnitude of 17.1 . We inspected archival and proprietary XMM-Newton X-ray observations of the intersection of the GRB error region and M31, obtained about 4 weeks prior to the outburst, in order to look for periodic variable X-ray sources. No SGR or anomalous X-ray pulsar (AXP) candidates (periods in the range 1-20 s) were detected. We discuss the possibility of detecting extragalactic SGRs/AXPs by identifying their periodic X-ray light curves. Our simulations suggest that the probability of detecting the periodic X-ray signal of one of the known Galactic SGRs/AXPs, if placed in M31, is about $10 \%$ using a $50 \mathrm{ks} X M M-N e w t o n$ exposure, increasing to $50 \%$ for a 2 Ms observation.
\end{abstract}

Subject headings: galaxies: individual (M31) — gamma rays: bursts — pulsars: general — stars: neutron — X-rays: individual (GRB 070201)

Online material: color figure, machine-readable table

\section{INTRODUCTION}

Soon after the discovery of gamma-ray bursts (GRBs), it was realized that some bursts repeat. The localization of these objects, called soft gamma-ray repeaters (SGRs), showed that they lie in the Local Group (Cline et al. 1980; Evans et al. 1980; Mazets \& Golenetskii 1981) and that their flare energy release ranges from $\sim 10^{39}$ to $10^{46}$ ergs.

In quiescence, SGRs (and also the related class of anomalous $\mathrm{X}$-ray pulsars, or AXPs) are detected as faint X-ray sources with luminosities in the range $\sim 10^{33}$ to $10^{36} \mathrm{ergs} \mathrm{s}^{-1}$. Their X-ray light curves are modulated with periodicities on the order of $10 \mathrm{~s}$ and period derivatives on the order of $10^{-10} \mathrm{~s} \mathrm{~s}^{-1}$. These properties suggest that SGRs are young neutron stars with ultrastrong magnetic fields $\left(\gtrsim 10^{14} \mathrm{G}\right)$. Contrary to "normal" neutron stars (i.e., radio pulsars), whose energy reservoir is rotational, SGRs' source of energy is most probably magnetic. The basic properties of SGRs are well explained by the popular magnetar model (Duncan \& Thompson 1992; Paczyński 1992).

\footnotetext{
1 Based on observations obtained with $X M M-N e w t o n$, an ESA science mission with instruments and contributions directly funded by ESA member states and NASA.

${ }^{2}$ Division of Physics, Mathematics and Astronomy, California Institute of Technology, Pasadena, CA 91125.

3 Max-Planck-Institut für extraterrestrische Physik, D-85740 Garching, Germany.

4 Physics Faculty, Weizmann Institute of Science, 76100 Rehovot, Israel.

5 Department of Astronomy and Astrophysics, Pennsylvania State University, University Park, PA 16802.

6 Jansky Fellow, National Radio Astronomy Observatory.

7 Department of Astronomy, University of Virginia, Charlottesville, VA 22904.

8 Kavli Institute for Astrophysics and Space Research, Massachusetts Institute of Technology, Cambridge, MA 02139.

9 Department of Physics and Institute of Astronomy, National Tsing Hua University, Hsinchu 30013, Taiwan.

${ }^{10}$ The Open University, Walton Hall, Milton Keynes MK7 6AA, UK.
}

Known SGRs and AXPs are associated with star-forming regions (for a review, see Gaensler et al. 2001). Moreover, some of them may be associated with supernova remnants (Cline et al. 1982; Rothschild et al. 1994; Hurley et al. 1999; Woods et al. 1999). However, Levan et al. (2006) have suggested a formation channel for magnetars in old stellar populations.

Unfortunately, only four SGRs are known to date, all in the Local Group (see Woods \& Thompson 2006 for a recent review), of which three reside in the Milky Way and one in the Large Magellanic Cloud. This small number of known SGRs severely hinders our ability to study their origin, environments (e.g., Gaensler et al. 2001), and rate of luminous flares (Palmer et al. 2005; Popov \& Stern 2006; Ofek 2007b).

However, the strongest SGR flares could be detected in nearby galaxies (e.g., Duncan 2001; Eichler 2002). Discovery of extragalactic SGRs is an exciting possibility that would enable us to enlarge the sample of known objects in this class. Unfortunately, extragalactic SGR flares have proved hard to recognize, and their observed rate of gamma-ray flaring will probably be on the order of several percent of the observed short-duration GRB rate (e.g., Lazzati et al. 2005; Nakar et al. 2006; Ofek 2007b). To date, only a small number of extragalactic SGR candidates are known: in M81 (Ofek et al. 2006; Frederiks et al. 2007b) and in NGC 6946 (Crider 2006). Unfortunately, each of these candidates has been observed to flare only once. Moreover, because of the limited positional accuracy of most current gamma-ray telescopes, they have astrometric uncertainties of hundreds of square arcminutes or more. This positional accuracy is too poor to allow environmental studies. Furthermore, given the relatively large positional uncertainty, it is possible that some of these candidates are due to chance coincidence.

Discovery of extragalactic SGRs will increase our statistical sample of such objects, and with accurate positions, it will be 


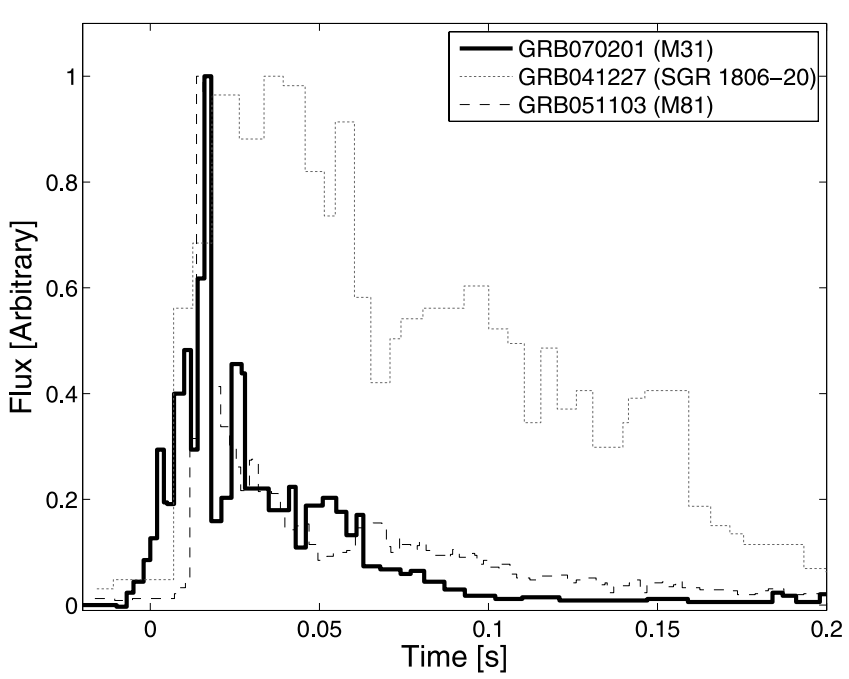

FIG. 1.-Konus-Wind gamma-ray light curve of GRB 070201 (heavy solid line), compared with the light curve of the 2004 December 27 SGR giant flare (dotted line) and the Konus-Wind light curve of GRB 051103 (dashed line; Ofek et al. 2006; Frederiks et al. 2007b). The light curve of the 2004 December 27 SGR giant flare is based on a digitization of Fig. 1 of Terasawa et al. (2005), while the light curve of GRB 070201 is based on a digitization of the $18-1160 \mathrm{keV}$ band light curve from the Konus-Wind Web site. The fluxes of the different bursts are scaled such they have the same peak flux. [See the electronic edition of the Journal for a color version of this figure.]

possible to study their environments. In particular, such discoveries may reveal a new population of SGRs that are not bound to star-forming regions (see, e.g., Levan et al. 2006).

In this paper, we discuss an extragalactic SGR giant-flare candidate associated with the nearby galaxy M31. At UTC 2007 February 1, 15:23:10.780, an intense short/hard GRB of $\sim 0.2 \mathrm{~s}$ duration was detected by the Interplanetary Network (IPN; see, e.g., Hurley et al. 1999). The burst was detected by Konus-Wind, the INTEGRAL SPI-ACS instrument, and the Swift Burst Alert Telescope $(B A T)^{11}$ (Golenetskii et al. 2007b), as well as by MESSENGER, while Suzaku and RHESSI were not able to observe the burst because of Earth occultation, and Odyssey was not able to observe it because of Mars occultation. Early on, Perley \& Bloom (2007) noted that the preliminary IPN annulus crossed the Andromeda galaxy (see also Ofek 2007a; Golenetskii et al. 2007a). Later, with the analysis of the MESSENGER data (Hurley et al. 2007) and reanalysis of the data (Mazets et al. 2008), the error region shrank to a $0.124 \mathrm{deg}^{2}$ quadrilateral that intersects M31.

The burst had the highest peak count rate of any GRB observed by Konus-Wind in 12 yr of operation (excluding Galactic SGRs). The GRB fluence in the Konus-Wind $20 \mathrm{keV}-1.2 \mathrm{MeV}$ band was $2.00_{-0.26}^{+0.10} \times 10^{-5} \mathrm{ergs} \mathrm{cm}^{-2}$, and its peak flux on a $2 \mathrm{~ms}$ timescale was $1.61_{-0.50}^{+0.29} \times 10^{-3} \mathrm{ergs} \mathrm{cm}^{-2} \mathrm{~s}^{-1}$ (90\% confidence; Mazets et al. 2008). The light curve, shown in Figure 1 (solid line; Mazets et al. 2008), had a "bumpy" rise with a timescale of $20 \mathrm{~ms}$ and two leading peaks with durations of a few milliseconds, while the decaying tail had a timescale of about $0.1 \mathrm{~s}$ (see discussion in Mazets et al. 2008).

Golenetskii et al. (2007a) and Mazets et al. (2008) found that the spectrum of GRB 070201 is well fitted by a power law with an exponential cutoff, $d N / d E \sim E^{-\alpha} \exp \left[-E(2-\alpha) / E_{p}\right]$, where $E$ is the energy. They found that for the first $64 \mathrm{~ms}$, the best-fit

\footnotetext{
11 The burst was outside the BAT coded field of view. Therefore, it was not localized by Swift.
}

parameters are $\alpha=0.52_{-0.15}^{+0.13}$ and $E_{p}=360_{-38}^{+44} \mathrm{keV}(90 \%$ confidence; $\chi^{2}=32$ for 35 degrees of freedom [dof ]), while the best-fit parameters for the time-integrated spectrum are $\alpha=0.98_{-0.11}^{+0.10}$ and $E_{p}=296_{-32}^{+38} \mathrm{keV}\left(\chi^{2} / \mathrm{dof}=40 / 40\right)$. Like GRB 070201, the spectrum of the 2004 December 27 giant flare of SGR 1806-20 at peak is not consistent with a blackbody spectrum but is well fitted by a power law with an exponential cutoff, with $\alpha=0.73_{-0.64}^{+0.47}$ $\left(\chi^{2} /\right.$ dof $=10.6 / 12$; Frederiks et al. 2007a).

Abbott et al. (2008) analyzed the available data from the Laser Interferometric Gravitational-Wave Observatory (LIGO; Abbott et al. 2007) collected within $180 \mathrm{~s}$ of the time of GRB 070201. They did not find any gravitational-wave source coincident with this GRB. Using these observations they rule out, at the $99 \%$ confidence level, origin in a compact binary merger (i.e., black holes or neutron stars) for this GRB with progenitor masses in the ranges $1 M_{\odot}<M_{1}<3 M_{\odot}$ and $1 M_{\odot}<M_{2}<40 M_{\odot}$, and at a distance below $3.5 \mathrm{Mpc}$.

In this paper, we present the case for GRB 070201 as a possible SGR giant flare in the nearby galaxy M31. In $\S 2$, we present our search for a visible-light transient associated with this GRB. We examine archival X-ray and UV images of the IPN error quadrilateral ( $(3)$, and we look for possible candidate pulsating X-ray sources that could be SGRs within M31 (§ 4). In $\S 5$, we quantify the probability of detecting X-ray pulsations of an SGR or AXP in M31, and finally we discuss the nature of GRB 070201 in $\S 6$.

\section{OBSERVATIONS}

Visible-light images of the Andromeda galaxy were obtained nightly by the $0.45 \mathrm{~m}$ ROTSE-IIIb telescope as part of the Texas Supernova Search (Quimby 2006). Routine unfiltered images covering the GRB error quadrilateral south of $\delta=+42^{\circ} 08^{\prime} 57^{\prime \prime}$ (J2000) (i.e., the southern $42 \%$ of the error box, including the intersection with the spiral arms) were taken on UTC 2007 February 2.0821, 10.6 hr after the GRB trigger.

We performed point-spread function-matched image subtraction on the data using a modified version of the Supernova Cosmology Project's search code (Perlmutter et al. 1999). After subtracting a reference template constructed from 37 ROTSE-IIIb images obtained between 2005 July and 2006 June, we found no new objects in the southern part of the error box covered, to a $5 \sigma$ limiting magnitude of 17.15 (calibrated against the USNO-B1.0 R2 magnitude; Monet et al. 2003). Assuming a distance to M31 of $770 \mathrm{kpc}$ (e.g., Ribas et al. 2005), and correcting for Galactic extinction in this direction (Schlegel et al.1998; Cardelli et al. 1989), this corresponds to an absolute magnitude limit of -7.4 .

\section{ARCHIVAL DATA}

The intersection region of the error quadrilateral of GRB 070201 with M31 has been observed by several facilities, including ROSAT, the Galaxy Evolution Explorer (GALEX), and XMM-Newton. The latter observed this field on several epochs, listed in Table 1. Analysis of the 2002 XMM-Newton data was presented by Pietsch et al. (2005). Interestingly, the last $X M M-N e w t o n$ observation of the field was obtained about 4 weeks prior to the GRB trigger, as part of an M31 XMM-Newton X-ray survey (Stiele et al. 2008). Source extraction from the $2007 X M M-N e w t o n$ images is presented in $\S 4$, while a complete catalog and analysis of the M31 observations will be presented in H. Stiele et al. (2008, in preparation).

In Figure 2, we present the GALEX near-UV image of the region of the error quadrilateral that intersects M31. In this figure, we show the refined (red lines; Mazets et al. 2008) and original 
TABLE 1

Log of XMM-Newton OBSERVATIONS

\begin{tabular}{|c|c|c|c|c|}
\hline Date & $\begin{array}{l}t_{\text {exp }} \\
(\mathrm{ks})\end{array}$ & $\begin{array}{l}\text { R.A. (J2000) } \\
\qquad(\mathrm{deg})\end{array}$ & $\begin{array}{l}\text { Decl. (J2000) } \\
\text { (deg) }\end{array}$ & $\begin{array}{l}\text { P.A. } \\
(\text { deg) }\end{array}$ \\
\hline 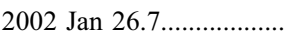 & 4 & 11.33543 & +41.93236 & 237.28 \\
\hline 2002 Jan $27.0 \ldots \ldots \ldots \ldots \ldots$ & 54 & 11.36929 & +41.92389 & 237.24 \\
\hline 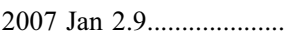 & 54 & 11.46008 & +41.51242 & 251.68 \\
\hline 2007 Jan $4.2 \ldots$ & 52 & 11.23317 & +42.14294 & 250.50 \\
\hline 2007 Jan $4.9 \ldots \ldots$ & 62 & 11.69142 & +41.88250 & 250.71 \\
\hline 2007 Jan $6.2 \ldots \ldots \ldots \ldots \ldots \ldots \ldots$ & 55 & 11.36483 & +41.91969 & 249.36 \\
\hline
\end{tabular}

NOTE.-List of XMM-Newton observations of the error quadrilateral of GRB 070201. P.A. is the position angle of the XMM-Newton instruments.

(blue lines; Hurley et al. 2007) IPN error quadrilateral, the ROSAT PSPC sources (blue diamonds; Supper et al. 2001), the XMMNewton sources detected in 2002 (yellow squares; Pietsch et al. 2005), the XMM-Newton sources detected in 2007 (red circles), and known candidate supernova remnants (cyan crosses; Magnier et al. 1995). The symbol size for the X-ray sources corresponds to their flux. We note that there is some overlap between the $X M M-N e w t o n$ observations. Therefore, having more than one symbol of the same type in almost the same position corresponds to a detection of the same source in the overlap regions between images taken the same year. The ROTSE-IIIb observations cover the error region south of the yellow line.

Several X-ray sources in the field of GRB 070201 (Fig. 2) show long-timescale variability between 2002 and 2007. Since several types of astrophysical X-ray sources are known to vary, this information by itself is not very constructive in the identification of an SGR X-ray counterpart in this field. However, an SGR or an AXP may reveal itself as a pulsating X-ray source with periodicity around $10 \mathrm{~s}$. In the next section we describe a search for such X-ray-variable sources. A thorough variability analysis of X-ray sources in the entire M31 galaxy will be presented in H. Stiele et al. (2008, in preparation).

\section{SEARCH FOR SHORT-PERIOD VARIABLE X-RAY SOURCES IN THE ERROR QUADRILATERAL}

All known SGRs and AXPs exhibit X-ray pulsations with periodicities in the range $2-12 \mathrm{~s}$. Therefore, it may be possible to

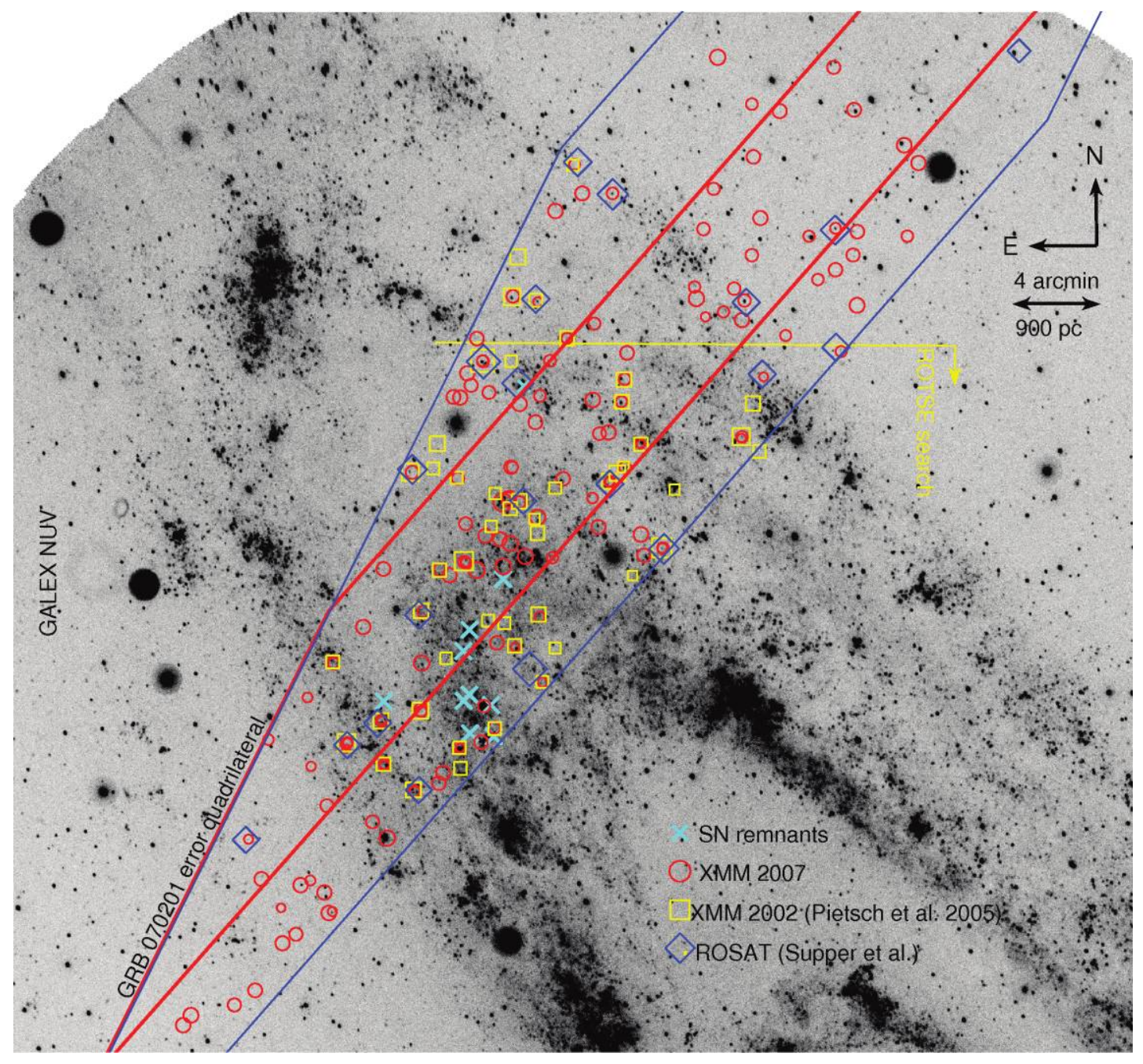

FIG. 2.-GALEX near-UV image, obtained on UTC 2003 September 5 (1940 s exposure), of the region of the error quadrilateral of GRB 070201 that intersects with M31.

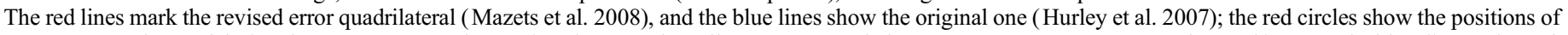

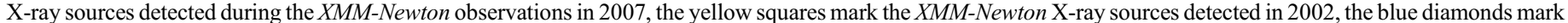

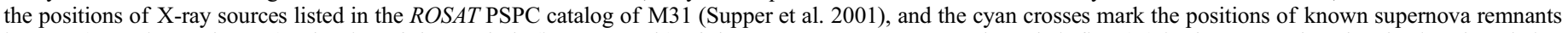

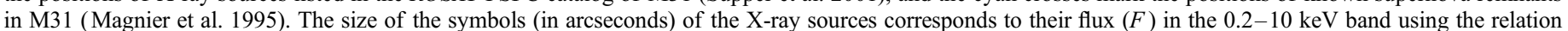

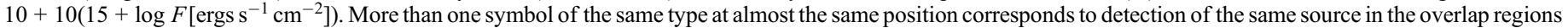
between images taken during the same year. The ROTSE-IIIb observations cover the entire error region south of the yellow line. 
TABLE 2

X-Ray Sources Searched for Periodicity

\begin{tabular}{|c|c|c|c|c|c|}
\hline Name & $\begin{array}{l}\text { R.A. (J2000) } \\
\qquad(\mathrm{deg})\end{array}$ & $\begin{array}{l}\text { Decl. (J2000) } \\
\text { (deg) }\end{array}$ & $\begin{array}{c}r^{\mathrm{a}} \\
(\operatorname{arcsec})\end{array}$ & Counts & ObsID/Detector \\
\hline $004603.5+414623 \ldots \ldots \ldots \ldots \ldots . .$. & 11.51444 & +41.77310 & 18.1 & 42274 & $0402561201 / \mathrm{PN}$ \\
\hline $004617.7+414258 \ldots \ldots \ldots \ldots \ldots \ldots$ & 11.57362 & +41.71622 & 29.5 & 24500 & 0402561201/PN \\
\hline 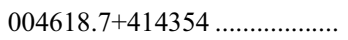 & 11.57812 & +41.73170 & 14.1 & 19633 & $0402561201 / \mathrm{PN}$ \\
\hline $004624.6+414414 \ldots \ldots \ldots \ldots \ldots \ldots$ & 11.60240 & +41.73723 & 18.3 & 22009 & $0402561201 / \mathrm{PN}$ \\
\hline $004625.6+414159 \ldots \ldots \ldots \ldots \ldots \ldots$ & 11.60687 & +41.69995 & 13.8 & 9300 & $0402561201 / \mathrm{PN}$ \\
\hline
\end{tabular}

Nоте.-Table 2 is published in its entirety in the electronic edition of the Astrophysical Journal. A portion is shown here for guidance regarding its form and content.

${ }^{a}$ Aperture radius in which source counts were extracted.

identify such objects in M31 by looking for X-ray-variable sources with periods in this range.

To look for such sources, we inspected the XMM-Newton pipeline-processed event files of the four fields observed in 2007 (Table 1) and removed time intervals during which particle events caused the event rate in the detector to flare by more than 2 standard deviations above the mean rate. We then created images of the $0.2-12 \mathrm{keV}$ events, binned to $4^{\prime \prime}$ resolution. For the purpose of identifying point sources, standard data selection was applied to make the images, to remove events near the edges of detector chips and bad pixels, and to reject events that were likely to be cosmic rays (pattern $>4$ for the pn, and pattern $>12$ for the MOS detectors). We then generated matching exposure maps and searched for point sources using the routine ewavelet separately for each detector. We extracted events for each source from the radius defined by ewavelet, which was $\approx 15^{\prime \prime}$. This radius contains about $50 \%$ of the photons for each source. The arrival times of the photons were transformed to the solar system barycenter using the tool barycen. Finally, we searched for periodicities in the extracted time-tagged photons using discrete fast Fourier transforms (FFTs). The time series were padded so that the numbers of points in the transforms were a power of 2 . This provides a frequency resolution slightly finer than $1 / t_{\text {exp }}$, where $t_{\exp }$ is the exposure time. The maximum frequency considered was the Nyquist frequency of the $13.6 \mathrm{~Hz}$ pn detector sampling rate, and the lowest frequency searched was $10^{-4} \mathrm{~Hz}$. We found no signals stronger than 19.15 times the mean of the power spectrum's noise. This cutoff power was selected such that the probability for a single source to surpass this threshold, in one or more of the $\sim 10^{6}$ tested frequencies, is about $\sim 1 \%$ (in the entire FFT-tested frequency range). Limiting ourself to the $1-20$ s periodicity range, this limit corresponds to a false-alarm probability of $\sim 0.05 \%$. In total we searched for periodicity among 149 X-ray sources within the original error quadrilateral (Hurley et al. 2007; Fig. 2, blue lines), which are listed in Table 2 . We did not find any periodic variable among the $X M M$-Newton X-ray sources.

\section{IS IT POSSIBLE TO DETECT THE MODULATED X-RAY EMISSION OF SGRs IN M31?}

The quiescent X-ray luminosity of known AXPs and SGRs ranges from $10^{33}$ to $10^{36} \mathrm{ergs} \mathrm{s}^{-1}$. At the distance of M31 (770 kpc; e.g., Ribas et al. 2005), these correspond to fluxes of $10^{-17}$ to $10^{-14} \mathrm{ergs} \mathrm{s}^{-1} \mathrm{~cm}^{-2}$ in the $2-10 \mathrm{keV}$ range. Given these flux levels, we discuss here the chances of detecting the modulated $\mathrm{X}$-ray light curves of SGRs or AXPs in M31 as a function of the flux of the X-ray source and its light-curve shape (i.e., the fraction of flux within a pulse). Specifically, we would like to answer the question, what is the probability of detecting an SGR or an AXP, based on its periodic X-ray signal, in the Andromeda galaxy? In order to answer this question, we performed the simulations described below.

In our simulations we assumed a $75 \mathrm{ks}$ exposure with the $X M M-N e w t o n$ fully depleted pn CCD, which roughly corresponds to a $50 \mathrm{ks}$ integration with all the European Photon Imaging Camera (EPIC) CCDs. Our simulated time-tagged X-ray light curves consist of the background expected for an $X M M$ Newton observation and a periodic signal. The periodic light curve consists of a nonvariable part and photons clumped in periodic pulses. In all the simulated photon-tagged light curves, the periodicity was set to exactly $10 \mathrm{~s}$ and the width of the periodic pulse was $20 \%$ of the period (i.e., $2 \mathrm{~s}$ ). We controlled the "shape" of the light curve by adjusting the fraction of photons within a pulse (hereafter the "pulse fraction").

We simulated light curves in a dense grid of count rates and pulse fractions. The count rates were set to be between $10^{-4}$ and $5 \times 10^{-2}$ counts s $^{-1}$ (along 100 logarithmically spaced grid points), with the pulse fractions in the range 0.21 to 0.81 (61 linearly spaced grid points). At each grid point, we simulated 100 photontagged light curves, and for each light curve we calculated the power spectrum and checked whether the $10 \mathrm{~s}$ period signal was stronger than 19.15 times the mean of the power spectrum noise. We note that this was the threshold used in the search for X-rayvariable sources described in $\S 4$. Finally, at each grid point we calculated the probability of recovering the periodic signal with a power exceeding the threshold, which corresponds to a falsealarm probability of about $1 \%$.

Figure 3 presents the results of these simulations. The contours show the probability of detecting X-ray periodicity with a false-alarm probability of $1 \%$ per source (assuming that for each source $10^{6}$ independent frequencies are tested), as a function of the two free parameters. The lower $X$-axis shows the observed count rate (with the luminosity at the distance of M31 on the upper axis), and the left $Y$-axis marks the fraction of energy within a pulse whose width is $20 \%$ of the period of the light curve. On the right $Y$-axis we show the rms pulsed fraction, $f_{\text {rms }}$, defined in Woods \& Thompson (2006; Table 14.2).

Next we compared these simulations with the actual properties of known AXPs and SGRs. For each of the 11 AXPs and SGRs listed in Woods \& Thompson (2006), for which the luminosity and rms pulse fraction $\left(f_{\text {rms }}\right)$ are known, we calculated the count rate (or range of count rates, in case of variables). We converted the luminosities of the AXPs/SGRs to count rates using the PIMMS Web tool ${ }^{12}$ and assumed a neutral hydrogen column density of $10^{21} \mathrm{~cm}^{-2}$ in the direction of M31 (Dickey \& Lockman 1990; Kalberla et al. 2005) and that the distance to M31 is $770 \mathrm{kpc}$ (e.g., Ribas et al. 2005). Furthermore, we assumed

${ }^{12}$ See http://cxc.harvard.edu/toolkit/pimms.jsp. 


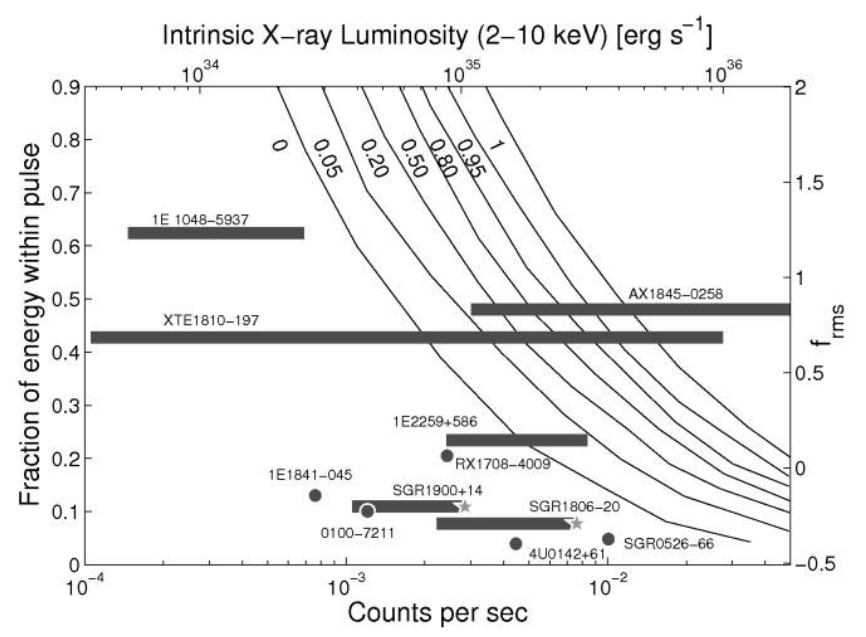

FIG. 3.- Probability (contours) of detecting, with $1 \%$ false-alarm probability, each of the Galactic AXPs/SGRs (in its quiescent state) if placed in the Andromeda galaxy, as a function the source count rate (lower $X$-axis) and luminosity (upper $X$-axis), and the fraction of energy within a $20 \%$ (of period) width pulse (left $Y$-axis). The translation of the energy within the pulse to rms pulse fraction, $f_{\mathrm{rms}}$ (for definition, see Table 14.2 of Woods \& Thompson 2006), is shown on the right $Y$-axis. The location of known AXPs/SGRs are shown as circles or lines (if variable). The simulations assume the $X M M$-Newton pn detector is observing the targets for $75 \mathrm{ks}$, which is roughly equivalent to the $50 \mathrm{ks} X M M$-Newton observations we analyzed. The properties of the SGRs/AXPs (i.e., luminosity range, spectral shape, and rms pulse fraction) were adopted from Woods \& Thompson (2006). To account for the observed elevated X-ray luminosity of SGRs about 1 month prior to giant flares, we increased the maximum quiescent luminosities of SGR 1900+14 and SGR 1806-20 by factors of 1.5 and 2, respectively (Woods et al. 2001; 2007). These elevated luminosities are marked with stars in the figure.

that the X-ray spectrum of each SGR/AXP is described only ${ }^{13}$ by a power law, and we adopted the measured power-law indices for each of these sources (Woods \& Thompson 2006). The location of the known SGRs and AXPs in pulse fraction versus $\mathrm{X}$-ray luminosity space (in the $2-10 \mathrm{keV}$ range ${ }^{14}$ are presented in Figure 3 as circles (or stripes to indicate a range).

The X-ray emission about 1 month before and several months after an SGR giant flare is known to be higher than "normal." This may elevate the probability of detecting X-ray emission from extragalactic SGR giant flares in the XMM-Newton M31 images taken 4 weeks prior to the burst. For example, the X-ray flux of SGR 1900+14 was about 1.5 times higher than normal, starting about 1 month prior to the SGR giant flare of 1998 August 27, and also for a year past the flare. In the case of the 2004 December 27 giant flare of SGR 1806-20, its X-ray emission was about 2 times brighter than the typical quiescent emission about 1 month prior to the burst. In Figure 3, we mark the elevated X-ray luminosities of SGR 1806-20 and SGR 1900+14 with stars.

Based on this plot, we estimate that the probability to detect a pulsating X-ray source associated with an AXP or SGR in M31, using the $50 \mathrm{ks} X M M$-Newton image we analyzed, is $\sim 10 \%$ (per SGR/AXP). We note however that for a 2 Ms exposure using $X M M-N e w t o n$, the probability to detect an AXP/SGR in M31 increases to $\sim 50 \%$.

\section{DISCUSSION}

In the following, we discuss the energetics and spectral and temporal properties of GRB 070201 ( $(6.1)$. Given the properties of this event, we then discuss its nature in $\S 6.2$.

\footnotetext{
13 Note that some of these objects have more complicated spectra.

14 The simulation assumes the observations are conducted in the $0.2-10 \mathrm{keV}$ band. For compatibility with Woods \& Thompson (2006), we present the luminosity in the $2-10 \mathrm{keV}$ band.
}

\subsection{Energetics, Spectrum, and Light Curve}

The IPN error quadrilateral of the bright GRB 070201 includes the outskirts of the nearby $(770 \mathrm{kpc})$ galaxy M31. Although it seems probable that this event is an SGR giant flare in M31, given the information at hand we cannot rule out the possibility that it was a short-duration GRB in the background. If indeed GRB 070201 originated in M31, the isotropic energy release from the burst, $1.41_{-0.18}^{+0.07} \times 10^{45} \mathrm{ergs}$, would be of the same order of magnitude as that emitted by SGR giant flares. For comparison, the isotropic energy release of the 1979 March 5 flare of SGR 0526-66 was over $6 \times 10^{44}$ ergs (Mazets et al. 1979) and that of the 1998 August 27 flare from SGR $1900+14$ was $2 \times 10^{44}$ ergs (Mazets et al. 1999), while the energy release from the 2004 December 27 giant flare from SGR $1806-20$ was as high as $(1-4) \times 10^{46} \mathrm{ergs}$ (Hurley et al. 2005; Palmer et al. 2005; Cameron et al. 2005).

In the context of the magnetar model for SGR giant flares (Thompson \& Duncan 1995, 1996), we expect the fireball to be optically thick and therefore to produce a quasi-thermal spectrum. As discussed in $\S 1$, the gamma-ray spectrum of GRB 070201 (Golenetskii et al. 2007a) at peak luminosity, as well as that of SGR 1806-20's 2004 December 27 giant flare (Frederiks et al. 2007a), is not well described by a blackbody spectrum. However, this does not necessarily mean that the spectrum of the burst is not a modified thermal spectrum. A simple consistency test for the SGR hypothesis is to assume the spectrum is quasi-thermal; we would then expect the blackbody radius of the emission region to be on the order of the radius of a neutron star. By approximating the gamma-ray spectrum of SGR flares as a blackbody spectrum, one can derive a rough blackbody radius for the bursting source. GRB 070201 had a peak luminosity (on a 2 ms timescale) of $1.14_{-0.35}^{+0.20} \times 10^{47} \mathrm{ergs} \mathrm{s}^{-1}$ and a peak energy of the observed gamma-ray spectrum corresponding to a blackbody temperature of $\sim 1.6 \times 10^{9} \mathrm{~K}$. Using the distance to M31, we find a blackbody radius of $60 \pm 40 \mathrm{~km}$. This radius is roughly consistent with the sizes derived for other SGR giant flares (e.g., Hurley et al. 2005; Ofek et al. 2006).

The temporal behavior of the gamma-ray emission from GRB 070201 (Fig. 1; see also Mazets et al. 2008) is somewhat different from that of the 2004 December 27, SGR 1806-20 giant flare (see, e.g., Hurley et al. 2005; Palmer et al. 2005; Terasawa et al. 2005). In GRB 070201, the rise to maximum flux is interrupted by two secondary peaks, and the total rise time is somewhat longer than in the case of the 2004 December 27 event. Moreover, it seems that the light curve of GRB 070201 is more variable than typical SGR giant-flare light curves. Such variability is consistent with that seen in the case of cosmological short-duration, hard-spectrum GRBs (e.g., Nakar \& Piran 2002; for a recent review, see Nakar 2007). However, our knowledge about SGR giant-flare light curves is based on a very small sample of events.

Given the data at hand, we cannot reject the possibility that GRB 070201 was a "genuine" short-duration GRB in the background. Most energetic short-duration GRBs have a total isotropic energy of $\sim 10^{51}$ ergs (e.g., Berger et al. 2007). If indeed $10^{51} \mathrm{ergs}$ is a rough upper limit for the energy of short GRBs, then we find that the luminosity distance to GRB 070201 is probably smaller than about $700 \mathrm{Mpc}(z \lesssim 0.15)$.

\subsection{The Nature of GRB 070201}

Given the short duration of this GRB and its spatial association with M31, there is a possibility that this burst was an SGR flare in M31. Estimating the probability for a chance coincidence is susceptible to the pitfalls of a posteriori statistics. Keeping this in mind, a rough estimate of the chance-coincidence probability 
is given by the sum of the area of M31 and the error quadrilateral of GRB 070201 (about $2 \mathrm{deg}^{2}$ ), multiplied by the number of short/ hard GRBs detected by Konus-Wind in the last 15 yr ( 30; Ofek $2007 \mathrm{~b}$ ), and divided by the area of the celestial sphere. This rough chance-coincidence probability is about $0.2 \%$. Therefore, we suggest that the simplest explanation is that GRB 070201 is indeed related to M31, and that it was an SGR giant flare. This is supported by the fact that, like other known SGRs (Gaensler et al. 2001), the GRB 070201 error box is spatially associated with star-forming regions in M31 (Fig. 2). However, our search and analysis do not rule out the possibility that GRB 070201 was a short-duration GRB in the background.

We note that if located in M31, this event's energy $\left(\sim 10^{45} \mathrm{ergs}\right)$ is too large for other kinds of known "Galactic GRBs" (e.g., Kasliwal et al. 2008). Moreover, Abbott et al. (2008) searched for a gravitational-wave signal coincident with the time of this burst using LIGO. The lack of signal argues against a compact-object merger (neutron stars or black holes) in M31, while it is consistent with this event's being an SGR giant flare in the Andromeda galaxy.

Finally, we note that instruments such as the Swift BAT (Gehrels et al. 2004) and the GLAST Burst Monitor (Band et al. 2004) will be able to detect fainter bursts, with energies of about $\sim 10^{42} \mathrm{ergs}$, from the Andromeda galaxy. Such bursts are several orders of magnitude more common than $\sim 10^{45}$ erg events. Therefore, with appropriate fast-response X-ray follow-up observations of GRBs with error regions that include nearby galaxies, it may be possible to detect the afterglows of such extragalactic SGR flares.

To summarize, we do not identify a visible-light afterglow associated with GRB 070201. Furthermore, we did not find any periodic X-ray source in archival XMM-Newton images of the intersection of the error quadrilateral of GRB 070201 with M31. We showed that the probability of detecting a pulsating X-ray source associated with an AXP or SGR in M31, in the available $X M M-N e w t o n$ data, is $\sim 10 \%$. Therefore, the fact that we did not find a pulsating $X$-ray source within the error quadrilateral does not rule out the possibility that GRB 070201 was an SGR giant flare in M31.

This work is supported in part by grants from NSF and NASA. H. S. acknowledges support from the Bundesministerium für Wirtschaft und Technologie/Deutsches Zentrum für Luft- und Raumfahrt (BMWI/DLR, FKZ 50 OR 0405). M. M. K. acknowledges the Moore Foundation for the George Ellory Hale Fellowship.
Abbott, B., et al. 2007, preprint (arXiv:0711.3041) 2008, ApJ, 681, 1419

Band, D., Briggs, M., Connaughton, V., Kippen, M., \& Preece, R. 2004, in AIP Conf. Proc. 727, Gamma-Ray Bursts: 30 Years of Discovery, ed. E. E. Fenimore \& M. Galassi (Melville, NY: AIP), 688

Berger, E., et al. 2007, ApJ, 664, 1000

Cameron, P. B., et al. 2005, Nature, 434, 1112

Cardelli, J. A., Clayton, G. C., \& Mathis, J. S. 1989, ApJ, 345, 245

Cline, T. L., et al. 1980, ApJ, 237, L1 1982, ApJ, 255, L45

Crider, A. 2006, in AIP Conf. Proc. 836, Gamma-Ray Bursts in the Swift Era, ed. S. S. Holt, N. Gehrels, \& J. A. Nousek (Melville, NY: AIP), 64

Dickey, J. M., \& Lockman, F. J. 1990, ARA\&A, 28, 215

Duncan, R. C. 2001, in AIP Conf. Proc. 586, Relativistic Astrophysics: 20th Texas Symposium, ed. J. C. Wheeler \& H. Martel (Melville, NY: AIP), 495 Duncan, R. C., \& Thompson, C. 1992, ApJ, 392, L9

Eichler, D. 2002, MNRAS, 335, 883

Evans, W. D., et al. 1980, ApJ, 237, L7

Frederiks, D. D., Golenetskii, S. V., Pal'shin, V. D., Aptekar, R. L., Ilyinskii, V. N., Oleinik, F. P., Mazets, E. P., \& Cline, T. L. 2007a, AZh Pisma, 33, 3 (English transl. Astron. Lett., 33, 1)

Frederiks, D. D., Pal'shin, V. D., Aptekar, R. L., Golenetskii, S. V., Cline, T. L., \& Mazets, E. P. 2007b, AZh Pisma, 33, 22 (English transl. Astron. Lett., 33, 19)

Gaensler, B. M., Slane, P. O., Gotthelf, E. V., \& Vasisht, G. 2001, ApJ, 559, 963

Gehrels, N., et al. 2004, ApJ, 611, 1005 (erratum 621, 558 [2005])

Golenetskii, S., Aptekar, R., Mazets, E., Pal'shin, V., Frederiks, D., \& Cline, T. 2007a, GCN Circ. 6094, http://gcn.gsfc.nasa.gov/gcn/gcn3/6094.gcn3

Golenetskii, S., et al. 2007b, GCN Circ. 6088, http://gcn.gsfc.nasa.gov/gen/gcn3/ 6088.gcn3

Hurley, K., Briggs, M. S., Kippen, R. M., Kouveliotou, C., Meegan, C., Fishman, G., Cline, T., \& Boer, M. 1999, ApJS, 120, 399 (erratum 125, 295)

Hurley, K., et al. 2005, Nature, 434, 1098

2007, GCN Circ. 6103, http://gen.gsfc.nasa.gov/gen/gen3/6103.gen3

Kalberla, P. M. W., Burton, W. B., Hartmann, D., Arnal, E. M., Bajaja, E., Morras, R., \& Pöppel, W. G. L. 2005, A\&A, 440, 775

Kasliwal, M. M., et al. 2008, ApJ, 678, 1127

Lazzati, D., Ghirlanda, G., \& Ghisellini, G. 2005, MNRAS, 362, L8

Levan, A. J., Wynn, G. A., Chapman, R., Davies, M. B., King, A. R., Priddey, R. S., \& Tanvir, N. R. 2006, MNRAS, 368, L1

Magnier, E. A., Prins, S., van Paradijs, J., Lewin, W. H. G., Supper, R., Hasinger, G., Pietsch, W., \& Trümper, J. 1995, A\&AS, 114, 215

\section{EFERENCES}

Mazets, E. P., Cline, T. L., Aptekar, R. L., Butterworth, P. S., Frederiks, D. D., Golenetskii, S. V., Il'inskii, V. N., \& Pal'shin, V. D. 1999, AZh Pisma, 25, 735 (English transl. Astron. Lett., 25, 635)

Mazets, E. P., \& Golenetskii, S. V. 1981, Ap\&SS, 75, 47

Mazets, E. P., Golenetskii, S. V., Il'inskii, V. N., Aptekar, R. L., \& Guryan, Yu. A. 1979, Nature, 282, 587

Mazets, E. P., et al. 2008, ApJ, 680, 545

Monet, D. G., et al. 2003, AJ, 125, 984

Nakar, E. 2007, Phys. Rep., 442, 166

Nakar, E., Gal-Yam, A., Piran, T., \& Fox, D. B. 2006, ApJ, 640, 849

Nakar, E., \& Piran, T. 2002, MNRAS, 330, 920

Ofek, E. O. 2007a, GCN Circ. 6092, http://gcn.gsfc.nasa.gov/gen/gcn3/6092.gcn3 2007b, ApJ, 659, 339

Ofek, E. O., et al. 2006, ApJ, 652, 507

Paczyński, B. 1992, Acta Astron., 42, 154

Palmer, D. M., et al. 2005, Nature, 434, 1107

Perley, D. A., \& Bloom, J. S. 2007, GCN Circ. 6091, http://gcn.gsfc.nasa.gov/ $\mathrm{gcn} / \mathrm{gcn} 3 / 6091 . \mathrm{gcn} 3$

Perlmutter, S., et al. 1999, ApJ, 517, 565

Pietsch, W., Freyberg, M., \& Haberl, F. 2005, A\&A, 434, 483

Popov, S. B., \& Stern, B. E. 2006, MNRAS, 365, 885

Quimby, R. 2006, Ph.D. thesis, Univ. Texas

Ribas, I., Jordi, C., Vilardell, F., Fitzpatrick, E. L., Hilditch, R. W., \& Guinan, E. F. 2005, ApJ, 635, L37

Rothschild, R. E., Kulkarni, S. R., \& Lingenfelter, R. E. 1994, Nature, 368, 432 Schlegel, D. J., Finkbeiner, D. P., \& Davis, M. 1998, ApJ, 500, 525

Stiele, H., et al. 2008, in Proc. ESAC Fac. Workshop on X-Rays from Nearby Galaxies, ed. S. Carpano, M. Ehle, \& W. Pietsch (MPE Rep. 295) (Garching: MPI extraterr. Phys.), 23

Supper, R., Hasinger, G., Lewin, W. H. G., Magnier, E. A., van Paradijs, J., Pietsch, W., Read, A. M., \& Trümper, J. 2001, A\&A, 373, 63

Terasawa, T., et al. 2005, Nature, 434, 1110

Thompson, C., \& Duncan, R. C. 1995, MNRAS, 275, 255

. 1996, ApJ, 473, 322

Woods, P. M., Kouveliotou, C., Finger, M. H., Göğüș, E., Wilson, C. A., Patel, S. K., Hurley, K., \& Swank, J. H. 2007, ApJ, 654, 470

Woods, P. M., Kouveliotou, C., Göğüss, E., Finger, M. H., Swank, J., Smith, D. A., Hurley, K., \& Thompson, C. 2001, ApJ, 552, 748

Woods, P. M., \& Thompson, C. 2006, in Compact Stellar X-Ray Sources, ed. W. H. G. Lewin \& M. van der Klis (Cambridge: Cambridge Univ. Press), 547

Woods, P. M., et al. 1999, ApJ, 519, L139 